Available on line at www.rac.es/racsam Mathematical Analysis

\author{
REVISTA DE LA REAL ACADEMIA DE CIENCIAS \\ EXACTAS, FISICAS Y NATURALES. \\ $\mathbb{S E R I E ~} \mathbb{A}: \mathbb{M} \mathbb{T} \mathbb{E} M \mathbb{A} \mathbb{T} \mathbb{C} \mathbb{S}$ \\ Madrid (España / Spain)
}

RACSAM 104 (1), 2010, 5-11. DOI:10.5052/RACSAM.2010.02

\title{
Barrelled spaces and mean ergodicity
}

\section{Krzysztof Piszczek}

\begin{abstract}
We characterize quasi-reflexive barrelled and complete locally convex Hausdorff spaces with a basis in terms of the properties of this basis. Moreover we prove that a complete, barrelled lcHs with a basis is quasi-reflexive of order one if and only if for every power bounded operator $T$, either $T$ or $T^{\prime}$ is mean ergodic.
\end{abstract}

\section{Espacios tonelados y ergodicidad en media}

Resumen. Se caracterizan los espacios localmente convexos Hausdorff casi-reflexivos, tonelados y completos con base en función de las propiedades de la base. Además se prueba que un espacio localmente convexo Hausdorff completo y tonelado con base es casi-reflexivo de orden 1 si y sólo si para cada operador $T$ de potencias acotadas, $T$ o $T^{\prime}$ es ergódico en media.

\section{Introduction}

In his very well known paper [9] R. C. James constructed an example of a non-reflexive Banach space $X$ such that its isometric embedding $\pi(X)$ into its second dual has a codimension 1 , that is $\operatorname{dim}\left(X^{\prime \prime} / \pi(X)\right)=$ 1. This led to the following generalization of reflexivity, introduced in [4]: a Banach space $X$ is called quasireflexive (of order $n$ ) if $\operatorname{codim}_{X^{\prime \prime}} \pi(X)<+\infty\left(\operatorname{codim}_{X^{\prime \prime}} \pi(X)=n\right)$. Properties of these spaces have been studied in $[5,8,10,19,20]$. It is well known that if $X$ is a barrelled and complete locally convex Hausdorff space (shortly $l c H s$ ) then $\pi(X)$ is a closed subset of $X^{\prime \prime}$, where $X^{\prime \prime}$ denotes the bidual of $X$ endowed with the strong topology. Therefore we say that a barrelled and complete lcHs is quasi-reflexive if the codimension of $\pi(X)$ in $X^{\prime \prime}$ is finite. Since every Banach space is barrelled and complete and we have an example of James' quasi-reflexive space $J$ then giving examples of barrelled and complete quasi-reflexive spaces is rather easy: for every reflexive barrelled and complete space $Y$ the space $Y \times J$ is quasi-reflexive. In [15] there are also examples of barrelled and complete quasi-reflexive spaces without Banach subspaces of infinite dimension.

Submitted by José Bonet

Received: November 2, 2009. Accepted: December 2, 2009

Keywords: Barrelled space, Schauder basis, mean ergodic operator, power bounded operator

Mathematics Subject Classifications: 46A04, 47A35

(c) 2010 Real Academia de Ciencias, España 
The aim of this paper is to continue the research of the author started in [17]. The motivation comes from the very important and beautiful paper [7] by Fonf, Lin and Wojtaszczyk where the authors characterize mean ergodic operators in reflexive Banach spaces with bases. Their work is then continued in the Fréchet space setting by Albanese, Bonet and Ricker in [1]. The authors answer, in particular, a question posed almost 40 years ago by Kalton in [11, p. 265]. Namely they prove that a barrelled and complete lcHs with a basis is reflexive iff every basis is shrinking iff every basis is boundedly complete.

The paper is divided into four parts. In Section 2 we extend the characterization of quasi-reflexive spaces with bases to the class of barrelled and complete lcHs'. The methods are analogous to the ones used in [17] therefore we often omit the proofs. Section 3 is devoted to the duality theorems for quasi-reflexive Fréchet and (DF)-spaces. To do this we rely on the original Singer's approach for Banach spaces used in [20]. In Section 4 we characterize quasi-reflexive barrelled and complete lcHs' with bases in terms of mean ergodicity of power bounded operators acting on this space. This is done exactly as in [17] (compare also [7, Th. 5]).

The general reference for functional analysis is [14] and for ergodic theory we refer the reader to [13].

\section{Quasi-reflexive barrelled and complete IcHs' with bases}

Recall that the topology of a locally convex space is determined by the basis of zero neighbourhoods which may always be chosen to be absolutely convex and closed. We will often use this fact without explicit mentioning. For such a zero neighbourhood $U$ we denote by $p_{U}$ its Minkowski functional. If $X$ is a locally convex Hausdorff space then by $X^{\prime}$ we mean the linear space $X^{*}$ of all continuous and linear functionals with the strong topology $b\left(X^{*}, X\right)$. A basis in a locally convex Hausdorff space $X$ is a sequence $\left(x_{j}\right)_{j \in \mathbb{N}}$ of elements with the following property: for every $x \in X$ there exists a unique sequence $\left(\alpha_{j}\right)_{j}$ of scalars such that the series $\sum_{j} \alpha_{j} x_{j}$ converges to $x$ in the topology of $X$. The linear functionals $f_{j}(x):=\alpha_{j}$ form the associated sequence of coefficient functionals. If they all are continuous then $\left(x_{j}\right)_{j}$ is called a Schauder basis. Recall that every basis in an barrelled lcHs is always a Schauder basis. We will use in the sequel the following notation: the symbol $\pi$ will stand for the canonical embedding of a locally convex Hausdorff space $X$ into its topological bidual $X^{\prime \prime}$ and for a Schauder basis $\left(x_{j}\right)_{j}$ in $X$ we will denote by $\left[f_{j}\right]$ the closed linear subspace of $X^{\prime}$ spanned by the associated sequence $\left(f_{j}\right)_{j}$ of coefficient functionals. Moreover by $\mathcal{B C B}$ we denote the class of all barrelled and complete locally convex Hausdorff spaces with a Schauder basis.

In [17] we prove the following result which, for Banach spaces, is due to Singer [20, Th. 3].

Theorem 1 A Fréchet space $X$ with a basis $\left(x_{j}\right)_{j}$ is quasi-reflexive of order $n$ if and only if there exists an integer $k, 0 \leq k \leq n$, such that the basis $\left(x_{j}\right)_{j}$ is $(n-k)$-boundedly complete and $k$-shrinking.

The aim of this Section is to extend this result onto $\mathcal{B C B}$ spaces. It turns out that the original proof uses the fact that a Fréchet space is barrelled and complete and therefore may be easily adapted to our setting. Consequently we present only those modifications which are necessary.

Theorem 2 Let $X$ be a $\mathcal{B C B}$ space and $\pi: X \hookrightarrow X^{\prime \prime}$ the canonical embedding. Let $\phi: X \rightarrow\left[f_{j}\right]^{\prime}$ be the map defined by $\phi(x):=\left.\pi(x)\right|_{\left[f_{j}\right]}$. The space $\phi(X)$ is a closed subspace of $\left[f_{j}\right]^{\prime}$ and $\pi(X) \oplus\left[f_{j}\right]^{\perp}$ is a closed subspace of $X^{\prime \prime}$.

SKETCH OF THE PROOF For arbitrary zero neighbourhoods $U, V$ we denote $F:=\left[f_{j}\right]$ and define numbers:

$$
\begin{aligned}
r_{U, V} & :=\sup \left\{t>0: t U^{\circ} \subset{\overline{V^{\circ} \cap F}}^{\sigma\left(X^{\prime}, X\right)}\right\}, \\
s_{U, V} & :=\inf _{p_{U}(x) \neq 0} \sup _{f \in V^{\circ} \cap F}\left|f\left(\frac{x}{p_{U}(x)}\right)\right|, \\
R_{U, V} & :=\sup \left\{p_{U}(x): x \in \bar{V}^{\sigma(X, F)}\right\}
\end{aligned}
$$


and proceed exactly as in the proof of [17, Th. 1].

The definitions below of a $k$-boundedly complete and a $k$-shrinking basis in a $\mathcal{B C B}$ space are analogous to the ones in Banach spaces, given in [20]. Let $X$ be a $\mathcal{B C B}$ space with a basis $\left(x_{j}\right)_{j \in \mathbb{N}}$. We define the following linear space of sequences of scalars

$$
\mathcal{X}:=\left\{\left(a_{j}\right)_{j}:\left(\sum_{j=1}^{m} a_{j} x_{j}\right)_{m=1}^{+\infty} \text { is bounded }\right\} .
$$

By [17, Lemma 2] the map

$$
J:\left[f_{j}\right]^{\prime} \rightarrow \mathcal{X}, \quad J(W):=\left(W\left(f_{j}\right)\right)_{j}
$$

is a linear isomorphism therefore we endow $\mathcal{X}$ with the topology inherited from $\left[f_{j}\right]^{\prime}$.

Definition 1 Let $X$ be a $\mathcal{B C B}$ space with a basis $\left(x_{j}\right)_{j}$. We shall say that this basis is $k$-boundedly complete if:

(i) in every $(k+1)$-dimensional subspace of $\mathcal{X}$ there exists a non-zero element $\left(a_{j}\right)_{j}$ such that the series $\sum_{j=1}^{+\infty} a_{j} x_{j}$ is convergent;

(ii) there exists $a(k+1)$-dimensional subspace of $\mathcal{X}$ for which the above element $\left(a_{j}\right)_{j}$ is unique up to a homothety.

Remark 1 For $k=0$ we obtain the definition of a boundedly complete basis which is due to James (see [9]) for Banach spaces and Dubinsky and Retherford (see [6] and [18]) for locally convex Hausdorff spaces.

Theorem 3 Let $X$ be a $\mathcal{B C B}$ space with a basis $\left(x_{j}\right)_{j}$, let $\left[f_{j}\right]$ be the closed subspace of $X^{\prime}$ spanned by the sequence $\left(f_{j}\right)_{j}$ of coefficient functionals and let $k$ be a non-negative integer. The following conditions are equivalent:

(1) $\left(x_{j}\right)_{j}$ is $k$-boundedly complete.

(2) $\operatorname{codim}_{X^{\prime \prime}}\left(\pi(X) \oplus\left[f_{j}\right]^{\perp}\right)=k$.

(3) $\operatorname{codim}_{\left[f_{j}\right]^{\prime}} \phi(X)=k$, where $\phi(x):=\left.\pi(x)\right|_{\left[f_{j}\right]}$.

Definition 2 Let $X$ be a $\mathcal{B C B}$ space with a basis $\left(x_{j}\right)_{j}$. We shall say that this basis is $k$-shrinking if:

(i) in every $(k+1)$-dimensional subspace of $X^{\prime}$ there exists a non-zero element $f$ such that

$$
\lim _{n \rightarrow+\infty} \sup _{x \in B \cap\left[x_{n+1}, x_{n+2}, \ldots\right]}|f(x)|=0
$$

for every bounded set $B \subset X$.

(ii) there exists a $(k+1)$-dimensional subspace of $X^{\prime}$ for which the above element $f$ is unique up to a homothety.

Remark 2 For $k=0$ we obtain the definition of a shrinking basis which is due to James (see [9]) for Banach spaces and Dubinsky and Retherford (see [6] and [18]) for locally convex Hausdorff spaces.

Theorem 4 Let $X$ be a $\mathcal{B C B}$ space with a basis $\left(x_{j}\right)_{j}$, let $\left[f_{j}\right]$ be the closed subspace of $X^{\prime}$ spanned by the sequence $\left(f_{j}\right)_{j}$ of coefficient functionals and let $k$ be a non-negative integer. The following assertions are equivalent:

(1) $\left(x_{j}\right)_{j}$ is $k$-shrinking. 
(2) $\operatorname{codim}_{X^{\prime}}\left[f_{j}\right]=k$.

The proof of the next result requires only linear isomorphisms of the considered spaces therefore is just a repetition of the proof of [17, Lemma 9].

Lemma 1 Let $X$ be a lcs and $V$ a total subspace of $X^{\prime}$. Then

$$
V^{\perp} \cap \pi(X)=\{0\} .
$$

If either $\operatorname{dim} V^{\perp}<+\infty$ or $\operatorname{codim}_{X^{\prime}} V<+\infty$ then

$$
\operatorname{dim} V^{\perp}=\operatorname{codim}_{X^{\prime}} V .
$$

If, in addition, $X$ is quasi-reflexive of order $n$, then:

$$
\begin{gathered}
0 \leq \operatorname{codim}_{X^{\prime}} V=\operatorname{dim} V^{\perp} \leq n, \\
0 \leq \operatorname{codim}_{X^{\prime \prime}}\left(\pi(X) \oplus V^{\perp}\right) \leq n, \\
\operatorname{codim}_{X^{\prime \prime}}\left(\pi(X) \oplus V^{\perp}\right)=n-\operatorname{codim}_{X^{\prime}} V .
\end{gathered}
$$

Now we are ready to state and prove the main result of this section which generalizes [17, Th. 10].

Theorem $5 \mathrm{~A} \mathcal{B C B}$ space $X$ is quasi-reflexive of order $n$ if and only if for every basis there exists an integer $k, 0 \leq k \leq n$, such that this basis is $(n-k)$-boundedly complete and $k$-shrinking.

Proof. Assume that $X$ is quasi-reflexive of order $n$. Since $\left[f_{j}\right]$ is a total subspace of $X^{\prime}$, by Lemma 1 we obtain

$$
n=\operatorname{codim}_{X^{\prime \prime}}\left(\pi(X) \oplus\left[f_{j}\right]^{\perp}\right)+\operatorname{codim}_{X^{\prime}}\left[f_{j}\right]
$$

and the conclusion follows by Theorems 3 (2) and 4 (2). If the converse holds, then by Theorem 3 (2) we have

$$
X^{\prime \prime}=\pi(X) \oplus\left[f_{j}\right]^{\perp} \oplus F
$$

with $\operatorname{dim} F=n-k$ and by Lemma 1

$$
\operatorname{dim}\left[f_{j}\right]^{\perp}=k .
$$

Therefore $\operatorname{codim}_{X^{\prime \prime}} \pi(X)=n$ and the proof is complete.

Corollary 1 A $\mathcal{B C B}$ space $X$ is quasi-reflexive if and only if every basis in $X$ is $k_{1}$-shrinking and $k_{2}$ boundedly complete for some non-negative integers $k_{1}, k_{2}$. In this case $X$ is quasi-reflexive of order $n=k_{1}+k_{2}$.

\section{Duality theorems.}

Recall that by [14, Prop. 23.23] every Fréchet space is barrelled which is not the case for arbitrary (DF)space. But if $X$ is a complete (DF)-space with a Schauder basis then by [16, Prop. 8.3.12] and [14, 23.21] $X$ is a $\mathcal{B C B}$ space. Therefore we may apply to such spaces results from the previous Section. The following theorems are generalizations of [20, Th. 4,5]. The proofs are analogous but at that same time short, therefore we include them below. All the bases are assumed to be Schauder.

Theorem 6 Let $X$ be a Fréchet space or a complete $(D F)$-space with a basis $\left(x_{j}\right)_{j}$. Denote by $F:=\left[f_{j}\right]$ the closed subspace of $X^{\prime}$ spanned by the sequence $\left(f_{j}\right)_{j}$ of coefficient functionals.

(1) $\left(x_{j}\right)_{j}$ is $k$-boundedly complete if and only if $\left(f_{j}\right)_{j}$ is a $k$-shrinking basis in $F$. 
(2) $\left(x_{j}\right)_{j}$ is $k$-shrinking if and only if $\left(f_{j}\right)_{j}$ is a $k$-boundedly complete basis in $F$.

Proof. (1) Define $w_{j}:=\phi\left(x_{j}\right)$ and observe that $\left(w_{j}\right)_{j}$ is a sequence of coefficient functionals for the basis $\left(f_{j}\right)_{j}$ in $F$. By Theorem $3\left(x_{j}\right)_{j}$ is $k$-boundedly complete if and only if $\operatorname{codim}_{F^{\prime}} \phi(X)=k$ while by Theorem $4\left(f_{j}\right)_{j}$ is $k$-shrinking if and only if $\operatorname{codim}_{F^{\prime}}\left[w_{j}\right]=k$. Here we have to know that $F$ is barrelled. In case $X$ is a (DF)-space, the space $F$ is clearly barrelled as a closed subspace of the Fréchet space $X^{\prime}$. In case $X$ is Fréchet, barrelledness of $F$ follows by [12, Th. 6.3]. Since by Theorem $2\left[w_{j}\right]=\phi(X)$, we are done.

(2) Since $\left[w_{j}\right]=\phi(X),\left(x_{j}\right)_{j}$ is $k$-shrinking in $X$ if and only if $\left(w_{j}\right)_{j}$ is $k$-shrinking in $\phi(X)$. By (1) (applied to the basis $\left(f_{j}\right)_{j}$ of $F$ ) this happens if and only if $\left(f_{j}\right)_{j}$ is $k$-boundedly complete in $F$.

Theorem 7 Let $X$ be Fréchet space or a complete (DF)-space. Suppose it is quasi-reflexive of order $n$ and let $0 \leq k \leq n$.

(1) X has a k-boundedly complete basis if and only if $X^{\prime}$ has a $k$-shrinking basis.

(2) X has a k-shrinking basis if and only if $X^{\prime}$ has a $k$-boundedly complete basis.

(3) X has a basis if and only if $X^{\prime}$ has a basis.

Proof. (1) If $\left(x_{j}\right)_{j}$ is a $k$-boundedly complete basis in $X$ then by Theorem 5 it is $(n-k)$-shrinking whence by Theorem $4 \operatorname{codim}_{X^{\prime}}\left[f_{j}\right]=n-k$. Consequently $X^{\prime}$ has a basis $\left(g_{i}\right)_{i}$ consisting of all $f_{j}$ 's together with suitable $n-k$ elements. Since by Theorem $6\left(f_{j}\right)_{j}$ is $k$-shrinking, the same is true for $\left(g_{i}\right)_{i}$. Assume now that $\left(f_{j}\right)_{j}$ is $k$-shrinking in $X^{\prime}$. By Theorem $4 \operatorname{codim}_{X^{\prime \prime}}\left[w_{j}\right]=k$, where $w_{i}\left(f_{j}\right)=\delta_{i j}$, and hence $X^{\prime \prime}$ has a basis $\left(v_{i}\right)_{i}$ consisting of all $w_{j}$ 's together with suitable $k$ elements. Since by Theorem 6 $\left(w_{j}\right)_{j}$ is $k$-boundedly complete, the same holds for $\left(v_{i}\right)_{i}$. By assumption $\operatorname{codim}_{X^{\prime \prime}} \pi(X)=n$ and all closed subspaces of codimension $n$ are isomorphic (the proof for arbitrary lcs' is analogous to [3]) therefore we can find an isomorphism $u$ : $\left[v_{i}\right]_{i=n+1}^{+\infty} \rightarrow \pi(X)$ and, consequently, $\left(\pi^{-1} \circ u\left(v_{i}\right)\right)_{i=n+1}^{+\infty}$ is a $k$-boundedly complete basis in $X$.

(2) By Theorem $5 X$ has a $k$-shrinking basis if and only if it has a $(n-k)$-boundedly complete basis. By (1) this happens if and only if $X^{\prime}$ has a $(n-k)$-shrinking basis and again by Theorem 5 if and only if $X^{\prime}$ has a $k$-boundedly complete basis.

(3) By Theorem $5 X$ has a basis if and only if it has a $k$-boundedly complete basis with $0 \leq k \leq n$. By (1) this happens if and only if $X^{\prime}$ has a $k$-shrinking basis and again by Theorem 5 if and only if $X^{\prime}$ has a basis.

\section{Quasi-reflexivity and mean ergodicity}

Recall that a continuous and linear operator $T$ on a locally convex space $X$ is called mean ergodic (see e.g. [13, Ch. $2, \S 2.1$, p. 73$])$ if the limits

$$
P x:=\lim _{n \rightarrow+\infty} \frac{1}{n} \sum_{k=1}^{n} T^{k} x
$$

exist for every element $x \in X$. It is called power bounded if the orbits $\left\{T^{k} x: k \in \mathbb{N}\right\}$ of all elements are bounded sets. If a space is barrelled this is equivalent to the equicontinuity of the set $\left(T^{k}\right)_{k} \subset L(X)$. If every power bounded operator is mean ergodic then the space $X$ is called mean ergodic. In $1931 \mathrm{~J}$. von Neumann proved that unitary operators on (complex) Hilbert spaces are mean ergodic and in 1938 F. Riesz showed that all $L^{p}$-spaces $(1<p<+\infty)$ are mean ergodic. A year later E. R. Lorch proved that reflexive Banach spaces are mean ergodic and the question arose whether the converse of Lorch's result is also true. In [7] the authors obtain a positive answer for Banach spaces with bases and also characterize finite 
dimensional Banach spaces by mean ergodicity of power bounded operators. These results have been extended onto the Fréchet space setting in [1] and to barrelled lcHs' in [2]. Our aim is to characterize quasi-reflexive $\mathcal{B C B}$ spaces in terms of mean ergodicity. Originally such a characterization is obtained for Banach spaces in [7, Th. 5] and later on extended by the author onto Fréchet spaces in [17, Th. 12].

The proof of the following result is similar to the one of [17, Th. 14] (compare [1, Section 3] and [21, Th. 1]) therefore we omit it.

Theorem 8 Let $k$ be a non-negative integer and let $X$ be a barrelled and complete lcHs which is not quasi-reflexive of order $k$. If $X$ has a $k$-shrinking basis then it has a $(k+1)$-shrinking basis as well.

The proof of the main result of this Section is similar to the original one given in [7] and its sketch is also presented in [17] therefore we state it without proof.

Theorem 9 Let $X$ be a non-reflexive $\mathcal{B C B}$ space. Then the following assertions are equivalent:

(1) $X$ is quasi-reflexive of order one,

(2) for every power bounded operator $T \in L(X)$, $T$ or $T^{\prime}: X^{\prime} \rightarrow X^{\prime}$ is mean ergodic.

Acknowledgement. The research of the author has been supported in the years 2007-2010 by the Ministry of Science and Higher Education, Poland, grant no. N N201 274033.

\section{References}

[1] Albanese, A. A.; Bonet, J. And Ricker, W. J., (2009). Mean ergodic operators in Fréchet spaces, Ann. Acad. Sci. Fenn. Math., 34, 401-436.

[2] Albanese, A. A.; Bonet, J., Ricker, W. J., On mean ergodic operators, In G .P. Curbera et. al. (Eds), Vector Measures, Integration and Related Topics, Proc. of Conf. on 'Vector Measures, Integration and Applications', Eichstätt, Sept. 2008, Birkhäuser Verlag, (to appear).

[3] Bessaga, C. And PeŁczyński, A., (1960). Banach spaces non-isomorphic to their Cartesian squares. I, Bull. Acad. Pol. Sci., 8, 2, 77-80.

[4] Civin, P. And Yood, B., (1957). Quasi-reflexive spaces, Proc. Amer. Math. Soc., 8, 906-911. DOI: 10.2307 12033689

[5] Cuttle, Y., (1961). On quasi-reflexive Banach spaces, Proc. Amer. Math. Soc., 12, 936-940. DOI: 10.2307 12034395

[6] Dubinsky, E. And Retherford, J. R., (1968). Schauder bases and Köthe sequence spaces, Trans. Amer. Math. Soc., 130, 265-280. DOI: 10.2307/1994971

[7] Fonf, V. P.; LIn, M. AND WoJTASZCZYK, P., (2001). Ergodic characterizations of reflexivity of Banach spaces, J. Funct. Anal., 187, 1, 146-162. DOI: 10.1006/jfan.2001.3806

[8] Godun, B. V., (1977). Equivalent norms on quasireflexive spaces with a basis (Russian), Dokl. Akad. Nauk Ukrain. SSR Ser. A, 9, 778-781, 863.

[9] JAMEs, R. C., (1950). Bases and reflexivity of Banach spaces, Ann. Math., 52, 3, 518-527. DOI: 10.2307 /1969430

[10] JAMES, R. C., (1977). Banach spaces quasi-reflexive of order one, Studia Math., 60, 2, 157-177.

[11] Kalton, N., (1970). Schauder bases and reflexivity, Studia Math., 38, 255-266.

[12] Kalton, N., (1970). Schauder decomposition in locally convex spaces, Proc. Cambr. Phil. Soc., 68, 2, 377-392. DOI: $10.1017 / \mathrm{S} 0305004100046193$ 
[13] Krengel, U., (1985). Ergodic theorems. With a supplement by Antoine Brunel, de Gruyter Studies in Mathematics, 6. Walter de Gruyter Co., Berlin.

[14] Meise, R. And Vogt, D., (1997). Introduction to Functional Analysis, Clarendon Press, Oxford.

[15] Maslyuchenko, V. K. And PlichKo, A. N., (1985). Quasireflexive locally convex spaces without Banach subspaces (Russian), Teor. Funktsii Funktsional. Anal. i Prilozhen, 44, 78-84; translation in J. Soviet Math., (1990), 48, 3, 307-312.

[16] Pérez Carreras, P. And Bonet, J., (1987). Barrelled Locally Convex Spaces, North-Holland Math. Stud., 131, Amsterdam.

[17] PiszczeK, K., (2010). Quasi-reflexive Fréchet spaces and mean ergodicity, J. Math. Anal. Appl., 361, 1, 224233. DOI: $10.1016 / j . j m a a .2009 .08 .060$

[18] Retherford, J. R., (1966). Bases, basic sequences and reflexivity of linear topological spaces, Math. Ann., 164, 3, 280-285. DOI: 10.1007/BF01360252

[19] Singer, I., (1963). On bases in quasi-reflexive Banach spaces, Rev. Math. Pures Appl., (Bucarest), 8, 2, 309-311.

[20] Singer, I., (1964). Bases and quasi-reflexivity of Banach spaces, Math. Ann., 153, 3, 199-209. DOI: 10.1007 /BF01360316

[21] ZipPIN, M., (1968). A remark on bases and reflexivity in Banach spaces, Israel J. Math., 6, 1, 74-79. DOI: 10.1007/BF02771607

\section{Krzysztof Piszczek}

Faculty of Mathematics and Computer Science, Adam Mickiewicz University, Poznań ul. Umultowska 87, 61-614 Poznań, Poland

kpk@amu.edu.pl 